\title{
Análises da Persistência na Lactação de Vacas da Raça Holandesa, Usando Produção no Dia do Controle e Modelo de Regressão Aleatória ${ }^{1}$
}

\author{
Jaime Araujo Cobuci ${ }^{2}$, Ricardo Frederico Euclydes ${ }^{3}$, Claudio Napolis Costa ${ }^{4}$, \\ Paulo Sávio Lopes ${ }^{3}$, Robledo de Ameida Torres ${ }^{3}$, Carmen Silva Pereira ${ }^{5}$
}

\begin{abstract}
RESUMO - Foram utilizados 87.045 registros de produção de leite, na primeira lactação, de 11.023 vacas da raça Holandesa, obtidos nos anos de 1997 a 2001, em diferentes rebanhos distribuídos em dez núcleos do Estado de Minas Gerais. Foram avaliados seis tipos de mensuração da persistência na lactação utilizando-se os valores genéticos da produção de leite, obtidos por meio do modelo de regressão aleatória - MRA. Utilizou-se a função de Wilmink na descrição dos efeitos aleatórios e fixos, pelo MRA. As estimativas de herdabilidade e de correlação genética, para as várias mensurações da persistência na lactação, variaram em decorrência da definição da persistência. As estimativas de herdabilidade para persistência na lactação variaram de 0,11 a 0,27 e as estimativas de correlação genética entre as mensurações da persistência na lactação e produção de leite até 305 dias, de -0,31 a 0,55, indicando que a persistência na lactação é uma característica de moderada herdabilidade e pouco correlacionada com a produção de leite até 305 dias. A seleção de animais para persistência na lactação, com o objetivo de alterar a forma da curva de lactação, pode ser eficiente.
\end{abstract}

Palavras-chave: correlação genética, herdabilidade, modelos de regressão aleatória, persistência na lactação, produção de leite no dia do controle, raça Holandesa

\section{Analysis of Persistency in the Lactation of Holstein Cows Using Test-Day Yield and Random Regression Model}

\begin{abstract}
A total of 87,045 milk yield records of 11,023 first-parity Holstein cows was utilized, obtained from 1997 to 2001 from different herds of 10 Minas Gerais locations. Six types of persistency measures in lactation were evaluated using milk yield breeding values, obtained by means of Random Regression Model - RRM. The Wilmink function was used to describe the random and fixed effects by RRM. Heritability estimates and genetic correlations for various persistency measures in lactation were dependent on the definition of persistency. The heritability estimates for persistency in lactation ranged from 0.11 to 0.27 and the genetic variations among persistency measures in lactation and milk yield up to d 305 ranged from -0.31 to 0.55 , showing that persistency in lactation is a trait of moderate heritability showing little correlation with milk yield up to $\mathrm{d} 305$. The selection of animals for persistency in lactation aiming to alter the lactation curve may be effective.
\end{abstract}

Key Words: genetic correlation, heritability, random regression model, persistency in lactation, test-day milk yield, Holstein cows

\section{Introdução}

Diferentes definições da persistência na lactação são encontradas na literatura. De forma geral, esta é definida como a capacidade da vaca em manter sua produção de leite após atingir a produção máxima na lactação.

Há, na literatura, quatro métodos de mensuração da persistência na lactação: 1) baseado em razões entre produção de leite em diferentes fases da lactação; 2) baseado na variação da produção de leite, ao longo da lactação; 3) baseado em parâmetros de modelos matemáticos; e 4) baseado nos valores genéticos obtidos por meio de coeficientes aleatórios dos modelos de regressão aleatória.

No entanto, o principal problema relativo ao estudo da persistência na lactação está no fato de como expressar a forma da curva de lactação em um único termo (Sölkner \& Fuchs, 1987). Muitas tentativas têm objetivado encontrar a melhor maneira de expressá-la (Sölkner \& Fuchs, 1987; Jamrozik etal., 1997b; Grossman et al., 1999; Jakobsen et al., 2002, entre outros).

\footnotetext{
1 Parte da tese apresentada à UFV, pelo primeiro autor, para obtenção do grau de "Doctor Scientiae" em Zootecnia.

2 Zootecnista, Pesquisador bolsista RD/CNPq. E.mail: jcobuci@cnpgl.embrapa.br

3 Professor da Universidade Federal de Viçosa-UFV, Viçosa, MG.

${ }^{4}$ Pesquisador da Embrapa Gado de Leite, Juiz de Fora, MG.

${ }^{5}$ Pesquisadora visitante da UFV/FAPEMIG.
} 
A persistência na lactação está diretamente relacionada com aspectos econômicos da atividade leiteira, pois a melhoria desta persistência pode contribuir para redução de custos no sistema de produção (Tekerli et al., 2000; Jakobsen et al., 2002).

Basicamente, há dois caminhos pelos quais os custos de produção podem ser reduzidos. O primeiro está associado à redução de custos com alimentação dos animais, em que parte da alimentação concentrada pode ser substituída pela alimentação volumosa (Sölkner \& Fuchs, 1987) e pela produção de leite adicional, obtida de animais com melhores níveis de persistência(Dekkers et al., 1996; Dekkers et al., 1998). O segundo está relacionado com redução de custos pela minimização de problemas associados à saúde e reprodução animal (Madsen, 1975; Sölkner \& Fuchs, 1987; Reents et al., 1996; Grossman et al., 1999).

De acordo com Sölkner \& Fuchs (1987), há indicativos da existência de diferenças genéticas, para persistência na lactação, entre animais, razão pela qual a seleção, para esta característica, possa ser vantajosa. Esta última afirmação também foi relatada nos trabalhos de Bar-Anan \& Ron (1985), Jamrozik et al. (1995), Swalve (1995a), Jamrozik et al. (1997b), Jamrozik et al. (1998) e Tekerli et al. (2000).

A utilização de modelos de regressão aleatória melhora a acurácia das avaliações genéticas e fornece um ótimo mecanismo para avaliar a persistência na lactação, pois, por meio desta metodologia, pode-se predizer o valor genético dos animais, em diferentes períodos da lactação (Lin \& Togashi, 2002).

Com o intuito de estudar a viabilidade da utilização da característica persistência na lactação em avaliações genéticas, em gado de leite, uma vez que são escassos os estudos sobre este assunto, principalmente em condições tropicais, objetivou-se, neste trabalho, estimar parâmetros genéticos para persistência na lactação, obtidos por diferentes tipos de mensuração, utilizando-se o método da máxima verossimilhança restrita e os modelos de regressão aleatória, bem como estimar correlações genéticas entre essas diferentes medidas de persistência na lactação e a produção de leite até 305 dias.

\section{Material e Métodos}

\section{Dados}

Os registros de produção de leite no dia do controle, utilizados neste estudo, referem-se à pri- meira lactação de 11.023 vacas da raça Holandesa, cujos partos ocorreram entre os anos de 1997 e 2001, em rebanhos distribuídos em dez núcleos do Estado de Minas Gerais. São provenientes do Serviço de Controle Leiteiro da Associação dos Criadores de Gado Holandês de Minas Gerais (ACGHMG) e compõem o Arquivo Zootécnico Nacional de Gado de Leite, gerenciado pela Embrapa Gado de Leite.

Com o intuito de se obter maior consistência dos dados, foram eliminadas as informações das vacas, filhas de reprodutores, que não tinham, no mínimo, quatro filhas por classe de rebanho-ano-mês do controle; vacas com idade menor que 18 e maior que 48 meses de idade no parto; lactações inferiores a 120 dias; registros de produção de leite diária inferior a $2,60 \mathrm{~kg}$ ou superior a 79,80 kg; e animais que não pertencessem às seguintes composições genéticas: puro de origem (PO) e igual ou superior a 31/32 Holandês. Satisfeitas todas as restrições, restaram 87.045 registros de 11.023 lactações de vacas, filhas de 936 touros pertencentes a 251 rebanhos, distribuídos no Estado de Minas Gerais.

O sumário das informações utilizadas neste estudo está apresentado na Tabela 1.

Os controles individuais de produção de leite foram agrupados em quatro classes de idade da vaca no parto, quais sejam, 20 a 24, 25 a 29, 30 a 34 e 35 a 48 meses, e em quatro estações de parto, janeiro a março, abril a junho, julho a setembro e outubro a dezembro. Esses grupos foram combinados e constituíram 16 classes de idade-estação de parto. Os grupos contemporâneos foram caracterizados pela

Tabela 1 - Sumário dos dados utilizados no estudo Table 1 - Summary of the informations used in the study

\begin{tabular}{lccc}
\hline $\begin{array}{l}\text { Núcleos } \\
\text { Locations }\end{array}$ & $\begin{array}{c}\text { Rebanhos } \\
\text { Herds }\end{array}$ & $\begin{array}{c}\text { Lactações } \\
\text { Lactations }\end{array}$ & $\begin{array}{c}\text { Controles } \\
\text { Test day }\end{array}$ \\
\hline Cruzília & 20 & 943 & 7.127 \\
Juiz de Fora & 35 & 887 & 6.834 \\
Lavras & 44 & 1.833 & 14.946 \\
Carmo do Rio Claro & 39 & 2.499 & 20.530 \\
Barbacena & 16 & 380 & 2.740 \\
Patrocínio & 16 & 674 & 5.170 \\
Uberlândia & 12 & 276 & 2.030 \\
Belo Horizonte & 53 & 3.012 & 23.398 \\
Itanhandu & 13 & 495 & 4.143 \\
Uberaba & 03 & 24 & 127 \\
\hline Total & 251 & 11.023 & 87.045 \\
\hline
\end{tabular}


combinação de rebanho, ano e mês em que foi realizado o controle leiteiro. Tanto as classes de idade da vaca-estação de parto como os grupos de animais contemporâneos foram considerados efeito fixo no modelo de regressão aleatória - MRA. Os coeficientes de regressão fixos, pelo MRA, foram estimados dentro das 16 classes de idade da vaca-estação de parto.

O arquivo de pedigree continha, no total, 19.918 animais, sendo identificados 8.537 vacas e 936 touros.

\section{Modelo de regressão aleatória}

Segundo Jamrozik et al. (1997a), o MRA tem sido indicado para avaliações genéticas com base na produção no dia do controle, devido à sua habilidade em separar a curva de lactação para cada animal, além de ser mais acurado e preciso que os modelos tradicionais.

Assim, a utilização dessa metodologia pode levar a estimativas mais acuradas dos efeitos genéticos e permanentes de ambiente, que afetam a persistência na lactação (Dekkers et al., 1998).

O modelo de regressão aleatória, aplicado aos registros de produção de leite no dia do controle, foi:

$$
y_{i j k l}=R A M C_{i}+\sum_{m=1}^{3} \beta_{k m} Z_{j l m}+\sum_{m=1}^{3} a_{j m} Z_{j l m}+\sum_{m=1}^{3} p_{j m} Z_{j l m}+e_{i j k l}
$$

em que $y_{i j k l}$ é o controle $l$ da vaca $j$, no período de dias em lactação $\left(\mathrm{t}_{\mathrm{j}}\right)$, dentro das classes $i$ (rebanho-anomês do controle) e $k$ (idade-estação de parto); $R A M C_{i}$, o efeito fixo de rebanho-ano-mês do controle; $e_{i j k l}$, 0 efeito aleatório residual associado a $y_{i j k l} ; \beta_{k m}$, o coeficiente de regressão fixo da produção de leite no dia do controle, em função de $t$, e descreve a forma da curva de lactação dentro das classes de idade da vaca-estação de parto; $a_{j m}$ e $p_{j m}$, os coeficientes de regressão aleatória que descrevem, respectivamente, os efeitos aleatórios genético e permanente de ambiente para cada animal; $Z_{j l m}$ é covariável representada pela função de Wilmink, em que $Z_{j 1 m}=\left(Z_{j 11} Z_{j 12} Z_{j 13}\right)^{\prime}=$ $(1 \mathrm{t} \mathrm{d})^{\prime}$, e d=exp ${ }^{-0,05 t}$; $t$ e $m$ são, respectivamente, o período de dias em lactação após o parto e o m-ésimo parâmetro da função.

A função de Wilmink (1987), utilizada na descrição dos efeitos genético e permanente de ambiente do MRA, é representada por

$$
y=a_{1}+a_{2} t+a_{3} \exp ^{(-0,05 t)}
$$

As soluções genéticas aditivas de cada animal $j$ são dadas pelo vetor $\left(\hat{a}_{1 j} \hat{a}_{2 j} \hat{a}_{3 j}\right)=\hat{a}_{j}^{\prime}$, entretanto, isoladamente, não são suficientes para classificar ou selecionar os animais. Todavia, funções dessas soluções fornecem importantes informações para os procedimentos de seleção. Assim, o valor genético aditivo esperado do animal $j$, no $t$-ésimo período de lactação, foi obtido por:

$$
V g_{j j}=\left[\hat{a}_{1 j}+\hat{a}_{2 j} \mathrm{t}+\hat{a}_{3 j} \mathrm{~d}\right]=\boldsymbol{Z}_{t}^{\prime} \hat{\boldsymbol{a}}_{j}
$$

O valor genético para produção de leite, considerando-se a lactação completa (em 305dias), $V g_{305}$, a partir da produção de leite no dia do controle, é obtido pela soma dos valores genéticos de cada dia em lactação (t) do animal $j$.

$$
V g_{305}=\sum_{t=6}^{305} V g_{t}=\left[\begin{array}{lll}
300 & 46650 & 15,1898
\end{array}\right] \hat{a}_{j}
$$

As análises consideraram os controles leiteiros realizados entre o 6 oㅡ e 305ํㅡㅁ dia de lactação após a data do parto; assim, devido à ausência dos controles leiteiros entre o $1^{\underline{0}}$ e $5 \underline{0}$ dia de lactação, o somatório foi restringido entre o 6o e 305음 dia de lactação.

\section{Persistência na lactação}

Do ponto de vista biológico, a curva de lactação apresenta três componentes, quais sejam, pico de produção, persistência na curva de lactação e duração do período de lactação (Ludwick \& Petersen, 1943). Segundo Wood (1967), entre esses componentes, a persistência é o mais importante.

A predição dos valores genéticos dos animais para as seis mensurações da persistência na lactação $\left(\mathrm{PS}_{\mathrm{i}}\right)$, utilizadas neste estudo, foi obtida pelas equações a seguir:

$$
P S_{1}=\left(V g_{280}-V g_{60}\right)=\left[\begin{array}{lll}
0 & 220 & -0,04979
\end{array}\right] \hat{a}_{j}
$$

A primeira medida da persistência na lactação, $\mathrm{PS}_{1}$, indica o diferencial genético na produção de leite (para mais ou para menos), no período que compreende o 60ํe e 280ํ dia de lactação, em relação à média dos animais com a mesma produção aos 60 dias de lactação, ou seja, é baseada na diferença das curvas de lactação dos animais, entre o $60^{\circ}$ e $280^{\circ}$ dia de lactação (Jamrozik et al., 1997b).

A segunda, $\mathrm{PS}_{2}$, é obtida pela diferença entre as áreas sob a curva de lactação, nos terços mediano e inicial da lactação (Jakobsen et al., 2002), dada por:

$$
P S_{2}=\left(\sum_{t=106}^{205} V g_{t}-\sum_{t=6}^{105} V g_{t}\right)=\left[\begin{array}{lll}
0 & 10000 & -14,98585
\end{array}\right] \hat{a}_{j}
$$

A terceira, $\mathrm{PS}_{3}$, é obtida pela área sob a curva de lactação, no terço final da lactação, subtraída pela 
área sob a curva, no terço inicial da lactação (Jakobsen et al., 2002), dada por:

$$
P S_{3}=\left(\sum_{t=206}^{305} V g_{t}-\sum_{t=6}^{105} V g_{t}\right)=\left[\begin{array}{lll}
0 & 20000 & -15,08682
\end{array}\right] \hat{a}_{j}
$$

A quarta, $\mathrm{PS}_{4}$, obtida pelo somatório das produções genéticas adicionais, no período do $61^{\circ}$ ao $280^{\circ}$ dia de lactação, como desvios da produção adicional aos 60 dias de lactação (Jamrozik et al., 1997b), é dada por:

$$
P S_{4}=\sum_{t=61}^{280}\left(V g_{t}-V g_{60}\right)=\left[\begin{array}{lll}
0 & 24310 & -9,98211
\end{array}\right] \hat{a}_{j}
$$

A quinta, $\mathrm{PS}_{5}$, obtida pelo somatório das contribuições de cada dia de produção genética adicional no período do $60^{\circ}$ ao 2790 dia de lactação, como desvios da produção adicional aos 280 dias de lactação (Jakobsen et al., 2002), é dada por:

$$
P S_{5}=\sum_{t=60}^{279}\left(V g_{t}-V g_{280}\right)=\left[\begin{array}{lll}
0 & -24310 & 1,02064
\end{array}\right] \hat{a}_{j}
$$

A sexta, $\mathrm{PS}_{6}$, é uma modificação da primeira medida da persistência na lactação (5), obtida pela diferença entre valores genéticos preditos para produção de leite aos $290^{\circ}$ e $90^{\circ}$ dia de lactação, dada por:

$$
P S_{6}=\left(V g_{290}-V g_{90}\right)=\left[\begin{array}{lll}
0 & 200-0,01111
\end{array}\right] \hat{a}_{j}
$$

Em geral, o pico de lactação das vacas, neste estudo, ocorreu ao redor dos 60 e 90 dias de lactação. Assim, a persistência na lactação foi obtida com base no período imediatamente seguinte ao pico de lactação, que compreende a produção de leite entre 90 e 290 dias após o início da lactação. Com isto, procurou-se, então, não utilizar as produções de leite referentes à primeira fase da lactação, visto que estas são mais influenciadas por fatores não-genéticos do que as outras fases da lactação. Segundo Ludwick \& Petersen (1943), a eliminação das primeiras semanas de produção de leite nas fórmulas de cálculo da persistência na lactação é indicada.

A área hachurada, na Figura 1, ilustra a diferença da persistência na lactação, dada por duas curvas de lactação, em que o ganho adicional na produção total de leite, obtido pelo maior nível de persistência na lactação, é dado pela área triangular, com base igual a 200 dias e altura dada pela diferença entre $\left(V g_{290}-V g_{90}\right)$.

Nota-se que os valores fenotípicos para cálculo da persistência na lactação não estão disponíveis neste estudo. Todos os resultados basearam-se nas expressões genéticas de PS, as quais são obtidas pela aplicação do MRA nas produções de leite no dia do controle.

\section{R. Bras. Zootec., v.33, n.3, p.546-554, 2004}

Ressalta-se que menores valores de $\mathrm{PS}_{1}, \mathrm{PS}_{2}$, $\mathrm{PS}_{3}, \mathrm{PS}_{4}$ e $\mathrm{PS}_{6}$ indicam maiores níveis de persistência na lactação, enquanto maiores valores de $\mathrm{PS}_{5}$ indicam maiores níveis de persistência na lactação.

\section{Estimação de parâmetros genéticos}

As matrizes que contêm as variâncias e covariâncias dos coeficientes aleotários foram obtidas por meio do programa REMLF90 (Misztal, 2001), que utiliza a metodologia de máxima verossimilhança restrita (REML) e o algoritmo de Maximização da Esperança (EM). O critério de convergência adotado foi de $1 \times 10^{-9}$.

As estimativas de variâncias genéticas $\hat{\sigma}_{a}^{2} \mathrm{e}$ permanentes de ambiente $\hat{\sigma}_{e p}^{2}$ para produção de leite em um período $t$ qualquer da lactação, são dadas por:

$$
\begin{aligned}
& \hat{\sigma}_{a}^{2}=z_{t}^{\prime} \hat{G} z_{t} \\
& \hat{\sigma}_{e p}^{2}=z_{t}^{\prime} \hat{P} z_{t}
\end{aligned}
$$

em que $\hat{G}$ e $\hat{P}$ são matrizes de variâncias e covariâncias entre os coeficientes de regressão aleatória; $z_{t}$ contém as covariáveis pertinente a um específico controle $l$, medido no tempo $t$ da lactação. Para as mensurações (ou medidas) da persistência na lactação, as variâncias são obtidas substituindo-se os vetores $z_{t}^{\prime}$ e $z_{t}$ pelos vetores $z_{i}^{\prime}$ e $z_{i}$ referentes ao

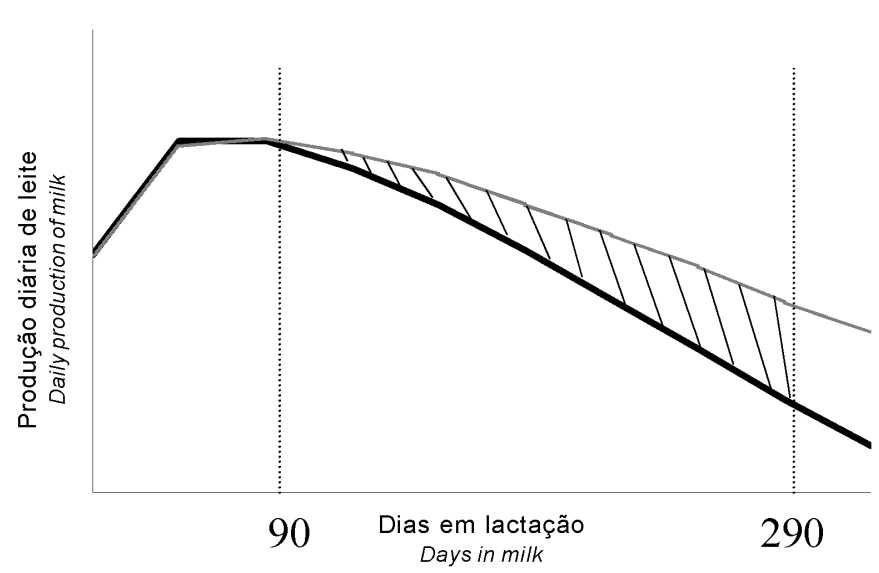

Figura 1 - Curvas de lactação de vacas com menor $(-)$ e maior persistência na lactação(-), em que o ganho adicional na produção de leite é dado pela área hachurada, ou seja, pelo diferencial no nível de persistência entre as curvas de lactação.

Figure1 - Lactation curves of cows with lower -) and higher persistency in lactation (-), where the additional gain in milk yield is given by the shaded area, in other words, for the differential in the persistency level among the lactation curves. 
i-ésimo tipo de mensuração da persistência na lactação (como descrito nas equações 5 a 10).

As estimativas de herdabilidade, para as diferentes mensurações da persistência na lactação $\left(\mathrm{PS}_{\mathrm{i}}\right)$ e para produção de leite até 305 dias de lactação (P305), foram calculadas, respectivamente, por:

$$
\hat{h}_{\left(P S_{i}\right)}^{2}=\frac{\hat{\sigma}_{a}^{2}(i)}{\hat{\sigma}_{a}^{2}(i)+\hat{\sigma}_{e p}^{2}(i)+\left(C_{i} * \hat{\sigma}_{e}^{2}\right)},
$$

$$
\hat{h}_{(P 305)}^{2}=\frac{\hat{\sigma}_{a}^{2}(P 305)}{\hat{\sigma}_{a}^{2}(P 305)+\hat{\sigma}_{e p}^{2}(P 305)+\left(300 * \hat{\sigma}_{e}^{2}\right)},
$$

em que $i=\mathrm{PS}_{1}, \mathrm{PS}_{2}, \mathrm{PS}_{3}, \mathrm{PS}_{4}, \mathrm{PS}_{5}$ e $\mathrm{PS}_{6}$ e $\mathrm{C}_{\mathrm{i}}$ é uma constante, dada pelos valores $2,200,200,48620$, 48620 e 2, respectivamente, para a i-ésima mensuração de persistência na lactação, $\mathrm{PS}_{1}, \mathrm{PS}_{2}$, $\mathrm{PS}_{3}, \mathrm{PS}_{4}, \mathrm{PS}_{5}$ e $\mathrm{PS}_{6}$.

$\mathrm{O}$ valor 300, que na equação (14) é representado por $C_{i}$ na equação (13), refere-se aos 300 dias de produção de leite inerentes ao intervalo do $6^{\mathrm{O}}$ ao $305^{\circ} \mathrm{O}$ dia de lactação, utilizado no cálculo da produção total de leite.

As estimativas de correlação genética entre as características $k$ e $m$ foram calculadas por:

$$
r \hat{g}_{\left(t^{\prime} t\right)}=\frac{\hat{\sigma}_{k m\left(t^{\prime} t\right)}}{\sqrt{\hat{\sigma}_{k k\left(t^{\prime}\right)}^{2} * \hat{\sigma}_{m m\left(t^{\prime} t\right)}^{2}}},
$$

em que $t^{\prime}=t$, se as observações consideradas referem-se ao mesmo período da lactação, e, se as observações forem de períodos diferentes.

\section{Resultados e Discussão}

\section{Persistência na lactação}

As matrizes de (co)variâncias dos coeficientes de regressão aleatória, atribuídas aos efeitos genético de animal e permanente de ambiente, estimadas por meio do modelo de regressão aleatória, são apresentadas na Tabela 2. A variância residual foi considerada constante $\left(7,166 \mathrm{~kg}^{2}\right)$, ao longo do período de lactação.

As estimativas de herdabilidade e as correlações genéticas e permanentes de ambiente, para as seis mensurações de persistência na lactação em vacas primíparas da raça Holandesa, encontram-se na Tabela 3.

Observa-se, na Tabela 3, que as estimativas de herdabilidade, para diferentes tipos de mensuração da persistência na lactação, variaram de 0,11 a 0,27. Embora os parâmetros genéticos sejam específicos da espécie, raça ou mesmo da população com a qual se está trabalhando, pode-se verificar certa concordância com as estimativas de herdabilidade relatadas por Gengler (1996), Jamrozik et al. (1998, 2000), Van Der Linde et al. (2000), Rekaya et al. (2001) e Jakobsen et al. (2002), as quais variaram de 0,09 a 0,30 e foram obtidas por meio de diferentes modelos de regressão aleatória e metodologia de estimação dos componentes de (co)variância. Contudo, foram bem superiores à relatada por Gengler et al. (1999), que encontraram herdabilidade igual a 0,05 .

A medida de persistência $\mathrm{PS}_{6}$ é uma modificação da $\mathrm{PS}_{1}$ e diferencia-se desta por ser calculada com base em diferentes períodos de lactação. Pode-se observar, na Tabela 3, que tanto as estimativas de herdabilidade quanto as de correlação genética mostraram-se diferenciadas nessas duas medidas de persistência. Tal fato pode ser atribuído à não-utilização do período que compreende a fase inicial da lactação em $\mathrm{PS}_{6}$.

Segundo Madsen (1975), diferenças entre herdabilidades para persistência na lactação podem ser causadas por três razões. A primeira está relacionada com a eficiência biológica do tipo de mensuração da persistência (ou seja, se as diferenças entre pro$\mathrm{t}^{\prime} \not \mathfrak{l}$ ções nos diferentes períodos devem ser consideradas em termos absolutos ou relativos). Entretanto, alguns tipos de mensuração podem ser melhores que

Tabela 2 - Estimativas de (co)variâncias genéticas $(\hat{G})$ e permanentes de ambiente $(\hat{P})$ para os coeficientes de regressão aleatória, obtidas pelo modelo de regressão aleatória

Table 2 - Estimates of genetic $(\hat{G})$ and permanent environmental $(\hat{P})$ (co)variance for random regression coefficients, obtained by random regression model

$\hat{G}=\left[\begin{array}{lll}\hat{g}_{11} & \hat{g}_{12} & \hat{g}_{13} \\ \hat{g}_{21} & \hat{g}_{22} & \hat{g}_{23} \\ \hat{g}_{31} & \hat{g}_{32} & \hat{g}_{33}\end{array}\right]=\left[\begin{array}{ccc}6,61 & -0,11 \times 10^{-1} & -6,70 \\ -0,11 \times 10^{-1} & 0,12 \times 10^{-3} & 0,96 \times 10^{-2} \\ -6,70 & 0,96 \times 10^{-2} & 15,59\end{array}\right]$
$\hat{P}=\left[\begin{array}{lll}\hat{p}_{11} & \hat{p}_{12} & \hat{p}_{13} \\ \hat{p}_{21} & \hat{p}_{22} & \hat{p}_{23} \\ \hat{p}_{31} & \hat{p}_{32} & \hat{p}_{33}\end{array}\right]=\left[\begin{array}{ccc}22,76 & -0,66 \times 10^{-1} & -19,79 \\ -0,66 \times 10^{-1} & 0,38 \times 10^{-3} & 0,57 \times 10^{-1} \\ -19,79 & 0,57 \times 10^{-1} & 54,26\end{array}\right]$

Os índices 1, 2 e 3 referem-se aos coeficientes de regressão aleatória $a_{1}, a_{2}$ e $a_{3}$, da função de Wilmink.

The indexes 1,2 and 3 refer to random regression coefficients $a_{1}, a_{2}$ and $a_{3}$, of the Wilmink function.

\section{R. Bras. Zootec., v.33, n.3, p.546-554, 2004}


outros, por expressarem, biologicamente, determinadas alterações na produção de leite durante a lactação. A segunda e a terceira estão associadas, respectivamente, à eficiência estatística do tipo de mensuração da persistência e à parte da lactação utilizada no cálculo da persistência na lactação. Assim, tipos de mensuração que excluem essas partes da lactação podem levar à obtenção de maiores valores de herdabilidade do que tipos que consideram as produções de leite em todo o período de lactação.

As herdabilidades e correlações genéticas para as várias mensurações da persistência na lactação dependem de como a persistência na lactação é definida.

Em geral, as estimativas de correlação genética foram altas, visto que apresentaram valores superiores a 0,86 (Tabela 3). Esses resultados foram superiores aos observados por Jakobsen et al. (2002), que encontraram correlações genéticas de $-0,41$ a 0,93 para cinco das seis mensurações de persistência avaliadas neste estudo.

As correlações permanentes de ambiente seguiram a mesma tendência e magnitude das correlações genéticas.

\section{Persistência na lactação e produção de leite}

As estimativas de herdabilidade para produção de leite, em diferentes períodos da lactação, produção de leite até 305 dias (P305), assim como as

Tabela 3 - Estimativas de herdabilidade (na diagonal) e correlações genéticas (acima da diagonal) e permanentes de ambiente (abaixo da diagonal) para as mensurações de persistência na lactação, obtidas pelo modelo de regressão aleatória

Table 3 - Estimates of heritabilities (on diagonal) and genetic correlations (above diagonal) and permanent environmental (below diagonal) for the persistency measures in lactation, obtained by the random regression model

\begin{tabular}{crrrrrr}
\hline $\begin{array}{c}\text { Característica } \\
\text { Trait }\end{array}$ & $\mathrm{PS}_{1}$ & $\mathrm{PS}_{2}$ & $\mathrm{PS}_{3}$ & $\mathrm{PS}_{4}$ & $\mathrm{PS}_{5}$ & $\mathrm{PS}_{6}$ \\
\hline $\mathrm{PS}_{1}$ & $\mathbf{0 , 1 5}$ & 0,89 & 0,98 & 0,99 & $-0,99$ & 0,99 \\
$\mathrm{PS}_{2}$ & 0,87 & $\mathbf{0 , 2 7}$ & 0,96 & 0,92 & $-0,86$ & 0,86 \\
$\mathrm{PS}_{3}$ & 0,97 & 0,95 & $\mathbf{0 , 2 6}$ & 0,99 & $-0,96$ & 0,96 \\
$\mathrm{PS}_{4}$ & 0,99 & 0,90 & 0,99 & $\mathbf{0 , 1 1}$ & $-0,99$ & 0,99 \\
$\mathrm{PS}_{5}$ & $-0,99$ & $-0,83$ & $-0,96$ & $-0,99$ & $\mathbf{0 , 1 1}$ & $-0,99$ \\
$\mathrm{PS}_{6}$ & 0,99 & 0,83 & 0,96 & 0,99 & $-0,99$ & $\mathbf{0 , 1 4}$ \\
\hline
\end{tabular}

$\mathrm{PS}_{1}$ a $\mathrm{PS}_{6}$ - persistência na lactação; P305 - produção de leite até 305 dias.

$P S_{1}$ a $P S_{6}$ - persistency in lactation; P305 - 305-day milk yield. correlações genéticas entre essas características e os seis tipos de mensuração da persistência na lactação, encontram-se na Tabela 4.

Pode-se verificar que os valores de correlações genéticas entre as várias medidas de persistência na lactação e P305 foram, de modo geral, baixos. No entanto, as medidas de persistência na lactação $\mathrm{PS}_{5}$ e $\mathrm{PS}_{6}$ apresentaram os menores valores de correlação genética com P305 (-0,31 e 0,31).

As correlações genéticas entre persistência na lactação e P305, encontradas neste estudo, foram superiores às relatadas por Jamrozik et al. (1998), Van Der Linde et al. (2000) e Jakobsen et al. (2002), em animais da raça Holandesa.

A análise dos resultados das correlações genéticas entre persistência e produção de leite, em determinados períodos da lactação (Tabela 4), indica associação negativa entre persistência na lactação e produção de leite na fase inicial da lactação (C6 e C30), ou seja, a melhoria do nível de persistência na lactação pode levar à redução da produção de leite nessa fase da lactação e, conseqüentemente, menores produções no pico de lactação, sem comprometer a produção total de leite.

Estudos relataram que vacas com alta produção até o pico de lactação apresentam declínio acentuado da produção de leite, quando comparadas às com menores produções, nessa primeira fase da lactação (Madsen, 1975; Lean et al., 1989). Vacas com menores taxas de declínios da produção (maior persistência) estão sujeitas a menores estresses fisiológicos, devido à ausência de elevadas produções no pico de lactação, minimizando, assim, a incidência de doenças de origem metabólica e problemas reprodutivos (Madsen, 1975; Sölkner \& Fuchs, 1987; Grossman et al., 1999; Tekerli et al., 2000).

$\mathrm{Na}$ Tabela 4, pode-se verificar que os valores das correlações genéticas entre persistência na lactação e produção de leite nos períodos selecionados da lactação são, de modo geral, bastante variados, indicando que a magnitude e a direção dessas estimativas dependem da definição de persistência utilizada. Verifica-se, ainda, existência de maiores correlações genéticas entre produções de leite, nos períodos selecionados da lactação e P305, do que entre as mensurações de persistência na lactação, as quais, com exceção da produção de leite no $6^{0}$ dia de lactação, apresentaram valores superiores a 0,80 .

Este resultado se assemelha ao observado por Jamrozik \& Schaeffer (1997) e Jakobsen et al. (2002),

R. Bras. Zootec., v.33, n.3, p.546-554, 2004 
que também encontraram menores valores para correlação genética entre controles leiteiros, realizados logo após o início da lactação, e produção total de leite.

$\mathrm{O}$ valor de 0,35 para herdabilidade da produção de leite até 305 dias (Tabela 4) foi semelhante aos relatados na literatura. Kettunen et al. (1997), Jamrozik \& Schaeffer (1997), Lidauer \& Mäntyssari (1999), Kettunen et al. (2000) e Jakobsen et al. (2002), ao utilizarem modelos de regressão aleatória, obtiveram valores para herdabilidade da produção de leite até 305 dias iguais a 0,$33 ; 0,32 ; 0,30 ; 0,36$ e 0,42 , respectivamente.

Ferreira (1999), ao utilizar também dados da Associação dos Criadores de Gado Holandês de Minas Gerais, cujas informações foram obtidas nos anos de 1989 a 1998, analisando as produções no dia do controle com modelos de características múltiplas, encontrou herdabilidade de 0,25 para a característica produção de leite até 305 dias.

Diferenças entre estimativas de herdabilidade, obtidas pelos modelos de regressão aleatória e pelos modelos que consideram a produção no dia do controle como medidas repetidas ou como características distintas, são relatadas na literatura (Kettunen et al., 1997). Essas divergências de resultados podem indicar necessidade de novas pesquisas, visando buscar funções matemáticas que melhor descrevam os efeitos genéticos e não-genéticos que atuam na produção de leite, por meio dos MRA.

Segundo Costa et al. (2002), a superestimação da herdabilidade tem sido um dos problemas associados ao ajuste da produção de leite no dia do controle, pelos modelos de regressão aleatória. Entretanto, a utilização apenas das informações de lactações completas em análises que envolvem modelos de regressão aleatória pode fornecer estimativas mais acuradas dos componentes de variância (Pool \& Meuwissen, 2000).

Na Tabela 5, são apresentadas as estimativas de correlação permanente de ambiente entre produção de leite em diferentes períodos da lactação, P305 e as várias mensurações de persistência na lactação. De forma geral, as correlações permanentes de ambiente entre as mensurações de persistência na lactação e P305 apresentaram valores baixos, o que condiz com as correlações de efeito permanente de ambiente, encontradas por Jamrozik et al. (1998).

Observa-se que, à exceção da produção de leite aos seis dias de lactação (C6), a correlação permanente de ambiente entre produção de leite na fase inicial (C30), mediana (C150) e final da lactação (C270) e P305 apresentou altos valores, próximos aos da unidade para produções na fase intermediária do período de lactação.

Ao comparar os resultados obtidos pelas seis mensurações de persistência na lactação avaliadas neste estudo, pode-se concluir que $\mathrm{PS}_{5}$ e $\mathrm{PS}_{6}$ são igualmente boas para serem escolhidas para uso em avaliações genéticas da persistência na lactação de vacas da raça Holandesa, no Estado de Minas Gerais.

A mensuração ideal para persistência na lactação deve apresentar valor econômico expressivo, elevada variância genética, alta herdabilidade e baixa correlação com a produção total de leite até 305 dias (Jakobsen et al., 2002).

\begin{tabular}{|c|c|c|c|c|c|c|c|c|}
\hline \multirow{2}{*}{$\begin{array}{r}\text { Table } 4- \\
\\
\\
\\
\text { la } \\
\text { Característic } \\
\text { Trait }\end{array}$} & \multicolumn{8}{|c|}{$\begin{array}{l}\text { Estimates heritabilities and genetic correlations among the measures of the } \\
\text { persistency in lactation and the production of milk in the selected periods of the } \\
\text { lactation and 305-day milk yield }\end{array}$} \\
\hline & $\mathrm{h}^{2}$ & $\mathrm{PS}_{1}$ & $\mathrm{PS}_{2}$ & $\mathrm{PS}_{3}$ & $\mathrm{PS}_{4}$ & $\mathrm{PS}_{5}$ & $\mathrm{PS}_{6}$ & P305 \\
\hline C6 & 0,15 & $-0,18$ & $-0,42$ & $-0,29$ & $-0,22$ & 0,15 & $-0,15$ & 0,24 \\
\hline C30 & 0,16 & $-0,23$ & $-0,05$ & $-0,16$ & $-0,20$ & 0,25 & $-0,25$ & 0,80 \\
\hline $\mathrm{C} 150$ & 0,24 & 0,30 & 0,53 & 0,41 & $-0,21$ & $-0,26$ & 0,26 & 0,99 \\
\hline $\mathrm{C} 270$ & 0,30 & 0,67 & 0,79 & 0,74 & 0,70 & $-0,64$ & 0,64 & 0,93 \\
\hline P305 & 0,35 & 0,35 & 0,55 & 0,44 & 0,38 & $-0,31$ & 0,31 & - \\
\hline
\end{tabular}

$\mathrm{h}^{2}$ - herdabilidade; $\mathrm{PS}_{1}$ a $\mathrm{PS}_{6}$ - persistência na lactação; P305 - produção de leite até 305 dias; C6 a C270 - produção de leite medida aos 6, 30,150 e 270 dias após o início da lactação. $h^{2}$ - heritability; $P S_{1}$ a $P S_{6}$ - persistency in lactation; P305 - 305-day milk yield; C6 a C270-milk yield at 6, 30, 150 e 270 days after the beginning of the lactation. 
Tabela 5 - Estimativas de correlação permanente de ambiente entre as mensurações da persistência na lactação e produção de leite nos períodos selecionados da lactação e produção de leite até 305 dias

Table 5 - Estimates of permanent environment correlations among the measures of persistency in lactation and production of milk in the selected periods of the lactation and 305day milk yield

\begin{tabular}{cccccccc}
\hline $\begin{array}{c}\text { Característica } \\
\text { Trait }\end{array}$ & $\mathrm{PS}_{1}$ & $\mathrm{PS}_{2}$ & $\mathrm{PS}_{3}$ & $\mathrm{PS}_{4}$ & $\mathrm{PS}_{5}$ & $\mathrm{PS}_{6}$ & $\mathrm{P} 305$ \\
\hline C6 & $-0,29$ & $-0,61$ & $-0,43$ & $-0,34$ & 0,24 & $-0,25$ & 0,35 \\
C30 & $-0,59$ & $-0,50$ & $-0,57$ & $-0,58$ & 0,59 & $-0,59$ & 0,80 \\
C150 & $-0,12$ & 0,11 & $-0,02$ & $-0,49$ & 0,14 & $-0,14$ & 0,99 \\
C270 & 0,51 & 0,61 & $-0,56$ & 0,53 & $-0,48$ & 0,48 & 0,83 \\
P305 & $-0,05$ & 0,12 & 0,02 & 0,03 & 0,07 & $-0,07$ & - \\
\hline
\end{tabular}

$\mathrm{PS}_{1}$ a PS $\mathrm{PS}_{6}$ - persistência na lactação; P305 - produção de leite até 305 dias; C6 a C270 - produção de leite medida aos 6, 30, 150 e 270 dias após o início da lactação.

$h^{2}$ - heritability; $P S_{1} a P S_{6}$ - persistency in lactation; P305 - 305-day milk yield; C6 a C270-milk yield at 6, 30, 150 e 270 days after the beginning of the lactation.

De acordo com Dekkers et al. (1996, 1998), a inclusão da persistência da lactação nas avaliações genéticas, em bovinos de leite, requer a estimação do valor econômico da característica persistência na lactação. Ao avaliarem os aspectos econômicos relacionados com persistência na lactação, esses autores relataram que o valor econômico dessa característica é influenciado pelos custos com alimentação, saúde e reprodução animal, assim como pelo retorno econômico obtido pela produção adicional de leite, devido à melhoria do nível de persistência na lactação dos animais.

Assim, a seleção de animais, em bovinos de leite, para maior persistência na lactação e, presumivelmente, para menores incidências de doenças e problemas reprodutivos, sem causar diminuição na produção total de leite, poderia ser feita com base nas mensurações $\mathrm{PS}_{5}$ e $\mathrm{PS}_{6}$, pois essas medidas apresentaram herdabilidades igualmente moderadas e foram pouco correlacionadas com a produção de leite até 305 dias.

\section{Conclusões}

A persistência na lactação é uma característica de moderada herdabilidade e de baixa correlação com a produção de leite até 305 dias, o que permite a seleção de animais com o objetivo de alterar o formato da curva de lactação.

Devem ser realizados estudos adicionais que visem encontrar funções matemáticas, que melhor descrevam as trajetórias dos efeitos genéticos e nãogenéticos que atuam na produção de leite e, conseqüentemente, na persistência na lactação.

\section{Agradecimento}

Os responsáveis por este trabalho agradecem ao professor Ignacy Misztal, da University of Georgia, EUA, por viabilizar a utilização do programa REMLF90.

\section{Literatura Citada}

BAR-ANAN, R.; RON, M. Associations among milk yield, yield persistency, conception, and culling of Israeli Holstein dairy cattle. Journal of Dairy Science, v.68, n.2, p.382-386, 1985.

COSTA, C.N.; MELO, C.M.R.; MACHADO, C.H.C. et al. Avaliação de funções polinomiais para ajuste da produção de leite no dia do controle de primeiras lactações de vacas Gir com modelo de regressão aleatória. In: REUNIÃO ANUAL DA SOCIEDADE BRASILEIRA DE ZOOTECNIA, 39., 2002, Recife. Anais... Recife: 2002. CD-ROM. Melhoramento Animal.

DEKKERS, J.C.M.; JAMROZIK, J.; TEN HAG, J.H. et al. Genetic and economic evaluation of persistency in dairy cattle. Interbull Bulletin, n.12, p.97-102, 1996.

DEKKERS, J.C.M.; TEM HAG, J.H.; WEERSINK, A. Economic aspects of persistency of lactation in dairy cattle. Livestock Production Science, v.53, p.237-252, 1998.

FERREIRA, W.J. Parâmetros genéticos para produção de leite no dia do controle de vacas da raça Holandesa. Viçosa, MG: Universidade Federal de Viçosa, 1999, 103p. Dissertação (Mestrado em Zootecnia) - Universidade Federal de Viçosa, 1999.

GENGLER, N. Persistency of lactation yields: a review. Interbull Bulletin, n.12, p.87-96, 1996.

GENGLER, N.; TIJANI, A.; WIGGANS, G.R. et al. Estimation of (co)variance functions of test day yields in first and later lactation of United States Holstein cows. Interbull Bulletin, n.22, p.69-73, 1999.

GROSSMAN, M.; HARTZ, S.M.; KOOPS, W.P. Persistency of lactation yield: A novel approach. Journal of Dairy Science, v.82, n.10, p.2192-2197, 1999.

R. Bras. Zootec., v.33, n.3, p.546-554, 2004 
JAKOBSEN, J.H.; MADSEN, P.; JENSEN, J. et al. Genetic parameters for milk production and persistency for Danish Holstein estimated in random regression models using REML. Journal of Dairy Science, v.85, n.6, p.607-1616, 2002.

JAMROZIK, J.; SCHAEFFER, L.R.; DEKKERS, J.C.M. Genetic evaluation of dairy cattle using test day yields and random regression model. Journal of Dairy Science, v.78, Suppl.1, p.249, 1995.

JAMROZIK, J.; KISTEMAKER, G.J.; DEKKERS, J.C.M. et al. Comparison of possible covariates for use in a random regression model for analyses of test day yields. Journal of Dairy Science, v.80, n.10, p.2550-2556, 1997a.

JAMROZIK, J.; SCHAEFFER. L.R.; DEKKERS, J.C.M. Genetic evaluation of dairy cattle using test day yields and random regression model. Journal of Dairy Science, v.80, n.6, p.1217-1226, 1997b.

JAMROZIK, J.; SCHAEFFER. L.R. Estimates of genetic parameters for a test day model with random regression for yield traits of first lactation Holstein. Journal of Dairy Science, v.80, n.4, p.762-770, 1997.

JAMROZIK, J.; JANSEN, G.; SCHAEFFER, L.R. et al. Analysis of persistency of lactation calculates from a random regression test day model. Interbull Bulletin, n.17, p.64-69, 1998.

JAMROZIK, J.; SCHAEFFER, R.L.; JANSEN, G.B. Approximate accuracies of prediction from random regression models. Livestock Production Science, v.66, p.85-92, 2000.

KETTUNEN, A.; MÄNTYSAARI, E. A.; STRADÉN, I. et al. Genetic parameters for test day milk yields of finish Ayrshires with random regression model. Journal of Dairy Science, v.80, Suppl. 1, p.197, 1997.

KETTUNEN, A.; MÄNTYSAARI, E.A.; POSO, J. Estimation of genetic parameters daily milk yield of primiparous Ayrshire cows by random regression test-day models. Livestock Production Science, v.66, p.251-261, 2000.

LEAN, I.J.; GALLAND, J.C.; SCOTT, J.L. Relationships between fertility, peak milk yields and lactational persistency in dairy cows. Theriogenology, v.31, n.5, p.1093-1103, 1989.

LIDAUER, M.; MÄNTYSAARI, E.A. Multiple trait reduced rank random regression test-day model for production traits. Interbull Bulletin, n.22, p.74-80, 1999.

LIN, C.Y.; TOGASHI, K. Simultaneous improvement of lactation milk and persistency. In: WORLD CONGRESS GENETIC APPLIED LIVESTOCK PRODUCTION, 7., 2002, Montpellier, France. Proceedings... Montpellier: Communication 09-39, CD-ROM, 2002.
LUDWICK, T.M.; PETERSEN, W.E. A measure of persistency of lactation of dairy cattle. Journal of Dairy Science, v.26, p.439-445, 1943.

MADSEN, O. A comparison of some suggested measures of persistency of milk yield in dairy cows. Animal Production, v.20, p.191-197, 1975.

MISZTAL, I. REMLF90 manual, 2001, ftp://nce.ads.uga.edu/ pub/ignacy/ blupf90/. Acessado em outubro de 2001.

POOL, M.H.; MEUWISSEN, T.H.E. Reduction of the number of parameters needed for a polynomial random regression test day model. Livestock Production Science, v.64, p.33-145, 2000.

REENTS, R.; REINHARDT, F.; ABRAMOWSKY, M. Calculation of persistency proofs form the German multilactation model for production traits. Interbull Bulletin, n.12, p.103-107, 1996.

REKAYA, R.; WEIGEL, K.A.; GIANOLA, D. Hierarchical nonlinear model for persistency of milk yield in the first three lactation of Holsteins. Livestock Production Science, v.68, p.81-187, 2001.

SÖLKNER, J.; FUCHS, W. A comparison of different measures of persistency with special respect to variation of Testday milk yields. Livestock Production Science, v.16, p.305-319, 1987.

SWALVE, H.H. Genetic relationship between dairy lactation persistency and yield. Journal of Animal Breeding Genetic, n.112, p.303-311, 1995a.

TEKERLI, M.; AKINCI, Z.; DOGAN, I. et al. Factors affecting the shape of lactation curves of Holstein cows from the Balikesir province of Tukey. Journal of Dairy Science, v.83, n.6, p.1381-1386, 2000.

Van Der LINDE, R. GROEN, A.; JONG, G. Estimation of genetic parameters for persistency of milk production in dairy cattle. Interbull Bulletin, n.25, p.113-116, 2000.

WILMINK, J.B.M. Adjustment of test-day milk, fat and protein yields for age, season and stage of lactation. Livestock Production Science, v.16, p.335-348, 1987.

WOOD, P.D.P. Algebric model of lactation curve in cattle. Nature, v.216, n.5111, p.164-165, 1967.

Recebido em: 10/12/02 Aceito em: 30/10/03 